\title{
Profinite rigidity for Seifert fibre spaces
}

\author{
Gareth Wilkes ${ }^{1}$
}

Received: 15 January 2016 / Accepted: 15 November 2016 / Published online: 29 November 2016

(C) The Author(s) 2016. This article is published with open access at Springerlink.com

\begin{abstract}
An interesting question is whether two 3-manifolds can be distinguished by computing and comparing their collections of finite covers; more precisely, by the profinite completions of their fundamental groups. In this paper, we solve this question completely for closed orientable Seifert fibre spaces. In particular, all Seifert fibre spaces are distinguished from each other by their profinite completions apart from some previously-known examples due to Hempel. We also characterize when bounded Seifert fibre space groups have isomorphic profinite completions, given some conditions on the boundary.
\end{abstract}

Keywords 3-Manifolds · Profinite rigidity $\cdot$ Seifert fibre spaces

Mathematics Subject Classification (2010) 57M05 · 57M27 · 20E18

\section{Introduction}

One possible algorithm to solve the homeomorphism problem for 3-manifolds could run as follows. Given two triangulated 3-manifolds $M_{1}$ and $M_{2}$, perform Pachner moves on $M_{1}$ to try to establish a homeomorphism with $M_{2}$. In parallel, compute a list of finite-sheeted covers of the two manifolds, along with regularity of the covers and the group of deck transformations. If at some covering degree a difference appears, the two manifolds will be shown to be non-homeomorphic.

The question arises, to what extent will this algorithm work? That is, could the collections of covers of two distinct 3-manifolds have the same structure? This is a manifestation of the wider question of when two groups have the same set of finite quotients. The naive statement in terms of sets of finite quotients is usually replaced with an equivalent formulation concerning

Gareth Wilkes

wilkes@maths.ox.ac.uk

1 Mathematical Institute, University of Oxford, Andrew Wiles Building, Radcliffe Observatory Quarter, Woodstock Road, Oxford OX2 6GG, UK 
the profinite completions of the two groups. The question is then one of 'profinite rigidity'. We make the following definition:

Definition 1.1 An (orientable) 3-manifold is profinitely rigid if the profinite completion distinguishes its fundamental group from all other fundamental groups of (orientable) 3manifolds.

In dimension 2, the analogous property is known to hold by work of Bridson et al. [6], who showed that the profinite completion distinguishes 2-orbifold groups not just from each other, but from all lattices in connected Lie groups.

For 3-manifolds, only a few examples are known to be profinitely rigid. Bridson and Reid [7] and Boileau and Friedl [5] have proved that the figure-eight knot group is profinitely rigid among 3-manifold groups, along with a handful of other knot groups. By contrast, there are large families known not to be profinitely rigid. Funar [11] built on work of Stebe [21] to give infinite families of Sol manifolds with the same finite quotients. Hempel [13] gave Seifert fibred families, with geometry $\mathbb{H}^{2} \times \mathbb{R}$.

These examples notwithstanding, the profinite completion of the fundamental group of a low-dimensional manifold is known to contain a large amount of information. For instance, Wilton and Zalesskii [24] have shown that the geometry (if any) of a 3-manifold is detected by the profinite completion. In particular, Seifert fibre spaces are distinguished from all other 3-manifolds. Lackenby [14] has shown that the pro-2 completion of a 3-manifold group determines whether that 3-manifold contains a pair of embedded surfaces which do not disconnect the manifold.

In this paper, we provide the full solution of the profinite rigidity question for closed orientable Seifert-fibred 3-manifolds. In effect, the above-cited examples of Hempel [13] are the only failures of profinite rigidity among these manifolds. The precise statement, when combined with the work in [24], is:

Theorem 1.2 Let $M$ be a (closed orientable) Seifert fibre space. Then either:

- $M$ is profinitely rigid; or

- $M$ has the geometry $\mathbb{H}^{2} \times \mathbb{R}$, is a surface bundle with periodic monodromy $\phi$ and the only 3-manifolds whose fundamental groups have the same finite quotients as $\pi_{1} M$ are the surface bundles with monodromy $\phi^{k}$, for $k$ coprime to the order of $\phi$.

The theorems of [24] are stated for closed manifolds, so we will be a little more circumspect about asserting profinite rigidity among all 3-manifolds. However we may still resolve the rigidity question among Seifert fibre spaces. For the precise statements see Theorems 5.8 and 5.9; in summary

Theorem 1.3 Let $M_{1}, M_{2}$ be Seifert fibre spaces with non-empty boundary. Then the following are equivalent:

- $\widehat{\pi_{1} M_{1}} \cong \widehat{\pi_{1} M_{2}}$, by an isomorphism inducing an isomorphism of peripheral systems.

- $M_{1}$ is a surface bundle with periodic monodromy $\phi$, and $M_{2}$ is a bundle over the same surface with monodromy $\phi^{k}$, where $k$ is coprime to the order of $\phi$.

The author would like to thank Marc Lackenby for suggesting this field of study and for many enlightening conversations during the development of this theorem. The author was supported by the EPSRC and by a Lamb and Flag Scholarship from St. John's College, Oxford. 
Remark 1.4 In this document, we will use the following conventions:

- All manifolds and orbifolds will be assumed compact, connected and without boundary unless otherwise stated; all 3-manifolds will be orientable;

- Abstract groups will be assumed finitely presented and will be denoted with Roman letters $G, H, \ldots$; they will be assumed to have the discrete topology.

- Profinite groups will be assumed topologically finitely generated and will be denoted with capital Greek letters $\Gamma, \Delta, \ldots$

- The symbols $\triangleleft_{\mathrm{f}}, \triangleleft_{\mathrm{o}}, \triangleleft_{p}$ will denote 'normal subgroup of finite index', 'open normal subgroup', 'normal subgroup of index a power of $p$ ' respectively; similar symbols will be used for not necessarily normal subgroups.

- There is a divergence in notation between profinite group theorists, who use $\mathbb{Z}_{p}$ to denote the $p$-adic integers, and manifold theorists for whom $\mathbb{Z}_{p}$ is usually the cyclic group of order $p$. To avoid any doubt, the cyclic group of order $p$ will be consistently denoted $\mathbb{Z} / p$ or $\mathbb{Z} / p \mathbb{Z}$.

\section{Cohomology of profinite groups}

\subsection{Goodness}

It will important to have control over the cohomology of profinite completions. We recall here certain theorems of this nature, which will be used freely without future reference. Serre [20] made the following definition:

Definition 2.1 A finitely generated group $G$ is good if for all finite $G$-modules $A$, the natural homomorphism

$$
H^{n}(\hat{G} ; A) \rightarrow H^{n}(G ; A)
$$

induced by $G \rightarrow \hat{G}$ is an isomorphism for all $n$.

Theorem 2.2 (Grunewald et al. [12]) All finitely generated Fuchsian groups are good.

Under certain finiteness assumptions which hold in our cases of interest, an extension of a good group by a good group is itself good (see [20]); furthermore, finite index subgroups of good groups are good. Hence:

\section{Corollary 2.3 The fundamental groups of Seifert fibre spaces are good.}

It will not be needed in the sequel, but it seems fitting to mention that in fact all 3-manifold groups are good. This theorem is of somewhat disputed attribution. It was proved by Wilton and Zalesskii [23] that a 3-manifold has good fundamental group if all pieces of its JSJ decomposition do. Seifert-fibred pieces are covered by the above corollary. That hyperbolic 3-manifold groups are good follows from the Virtually Compact Special Theorem and its various consequences. There are numerous ways one may deduce this; one may use the Virtual Fibring Theorem of Agol [1] or another route as outlined in [10]. In stating the Theorem as due to Agol, Wilton-Zalesskii, and Wise we aim to recognise those who contributed most. A full account and list of references may be found in [4].

Theorem 2.4 (Agol, Wilton-Zalesskii, Wise) Fundamental groups of compact 3-manifolds are good. 


\subsection{Chain complexes}

It will be necessary later to work with certain exact sequences of modules over the group ring of a profinite group. In this section we will recall and prove some of the necessary tools.

Definition 2.5 Given a profinite abelian group $A$ (usually $\hat{\mathbb{Z}}, \hat{\mathbb{Z}}_{(p)}$ or a finite abelian group) and a profinite group $\Gamma$, the completed group ring $A[[\Gamma]]$ is defined as the inverse limit

$$
\lim _{A^{\prime}, N} A / A^{\prime}[\Gamma / N]
$$

of group rings indexed over the finite index open normal subgroups $A^{\prime}, N$ of $A, \Gamma$. It is a compact Hausdorff topological ring.

Modules over $A[[\Gamma]]$, together with continuous module maps, form an abelian category with the same formal properties as the category of $R$-modules for a ring $R$; so the machinery of homological algebra works and we can define profinite group cohomology by starting from an arbitrary resolution of $\hat{\mathbb{Z}}$ by projective (left) $\hat{\mathbb{Z}}[[\Gamma]]$-modules and applying the functor $\operatorname{Hom}_{\hat{\mathbb{Z}}[[\Gamma]]}(-, M)$ giving the continuous homomorphisms from a module to $M$. If $M$ is a module with trivial $\Gamma$-action, we can factor this through the functor $\hat{\mathbb{Z}} \otimes_{\hat{\mathbb{Z}}[[\Gamma]]}$ - which 'forgets the $\Gamma$-action' on the chain complex.

We will need to show that, under certain conditions, a free resolution of $\mathbb{Z}$ by $\mathbb{Z}[G]$ modules yields a free resolution of $\hat{\mathbb{Z}}$ by $\hat{\mathbb{Z}}[[\hat{G}]]$-modules. To this end we use the following propositions and definitions, which are adapted from results in [15].

Definition 2.6 A discrete group $G$ is of type $\operatorname{FP}(n)$ if there is a resolution of the trivial module $\mathbb{Z}$ by projective $\mathbb{Z}[G]$-modules $P_{\bullet}$, such that $P_{i}$ is finitely generated for $0 \leq i \leq n$.

Proposition 2.7 Let $G$ be a discrete group which is good. Then:

- $\lim _{K \leq_{\mathrm{f}} G} H^{q}(K ; M)=0$ for every finite $G$-module $M$ and all $q \geq 1$

- If $G$ is of type $F P(n)$, then $\lim _{K \leq_{\mathrm{f}} G} H_{q}(K ; M)=0$ for every finite $G$-module $M$ and all $1 \leq q \leq n$.

Proof First note we may restrict to the case of trivial modules in the conclusions, as any finite $G$-module $M$ becomes trivial over $K$ for a cofinal subset of $\left\{K \leq_{\mathrm{f}} G\right\}$. Thus we may view $M$ interchangeably as a left or right module. The maps $\operatorname{res}_{K^{\prime}}^{K}: H^{q}(K ; M) \rightarrow H^{q}\left(K^{\prime} ; M\right)$ are given by restriction of cochains. The direct limit in question (categorically a colimit) is zero if all elements of $H^{q}(K ; M)$ are 'eventually zero'; that is, for all $x \in H^{q}(K ; M)$ there is some $K^{\prime} \leq K$ such that $x$ is mapped to zero under the restriction map $H^{q}(K ; M) \rightarrow H^{q}\left(K^{\prime} ; M\right)$. By goodness of $K$, there is a natural identification $H^{q}(K ; M) \cong H^{q}(\hat{K} ; M)$ so we may represent $x$ as a continuous cochain $\xi: \hat{K}^{q} \rightarrow M(q>0)$. The preimage of 0 under $\xi$ is some open subset of $\hat{K}^{q}$; products of open subgroups of $\hat{K}$ form a neighbourhood basis in $\hat{K}^{q}$, so we may choose $\Delta \leq_{\mathrm{o}} \hat{K}$ such that $\left.\xi\right|_{\Delta^{q}}=0$; then setting $K^{\prime}=K \cap \Delta$ (so that $\Delta=\hat{K}^{\prime}$ ) the commuting diagram

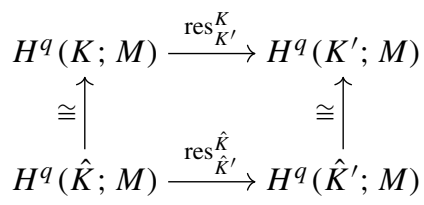

shows that $\operatorname{res}_{K^{\prime}}^{K}(x)=0$; hence that $\lim _{K \leq_{\mathrm{f}} G} H^{q}(K ; M)=0$. 
For the second conclusion, assume $G$ is of type $\operatorname{FP}(n)$. Then $H_{q}(K ; M)$ is finite for all $0 \leq q \leq n, K \leq_{\mathrm{f}} G$ and $M$ a finite $G$-module, and similarly for the cohomology. Recall that an inverse limit of finite abelian groups $A_{i}$ is trivial if and only if for each $i$ in the indexing set, there exists $j \geq i$ such that $A_{j} \rightarrow A_{i}$ is the zero map; and similarly for a direct limit of finite abelian groups.

So let $K$ be a finite index subgroup of $G$, and take $K^{\prime}$ such that the restriction map $\operatorname{res}_{K^{\prime}}^{K}$ is zero on each $H^{q}$. We show that we can dualise this to find that the corestriction map is also zero. Note that a finite-index subgroup of a group of type $\operatorname{FP}(n)$ is also of type $\operatorname{FP}(n)$. Let $P$. be a projective resolution of $\mathbb{Z}$ by left $\mathbb{Z} K$-modules, which is finitely generated in dimension at most $n$. There is a natural isomorphism (see [9], Proposition II.5.2)

$$
\operatorname{Hom}_{\mathbb{Z} K}\left(P_{\bullet}, \operatorname{Hom}_{\mathbb{Z}}(M, \mathbb{Q} / \mathbb{Z})\right) \cong \operatorname{Hom}_{\mathbb{Z}}\left(M \otimes P_{\bullet}, \mathbb{Q} / \mathbb{Z}\right)
$$

Now take homology; $\mathbb{Q} / \mathbb{Z}$ is an injective abelian group, so Hom $(-, \mathbb{Q} / \mathbb{Z})$ is an exact functor and commutes with homology; hence we get a natural isomorphism

$$
H^{q}\left(K ; M^{*}\right) \cong\left(H_{q}(K ; M)\right)^{*}
$$

where $N^{*}$ denotes the dual $\operatorname{Hom}(N, \mathbb{Q} / \mathbb{Z})$ of an abelian group. Finite abelian groups are isomorphic to their dual and canonically isomorphic to their double-dual; so we get a natural isomorphism

$$
H^{q}\left(K ; M^{*}\right)^{*} \cong H_{q}(K ; M)
$$

in dimensions $0 \leq q \leq n$ where the right hand side is finite. The inclusion $K^{\prime} \rightarrow K$ induces the zero map on the left hand side by assumption, noting that $M$ is isomorphic to $M^{*}$ so the restriction map with $M^{*}$ coefficients also vanishes. Hence the map on the right hand side, the corestriction map, is zero.

To prove the next proposition, we will need some exactness properties of the functor lim. In general this functor will not be exact and so will not commute with homology. A well-known condition for exactness is the Mittag-Leffler condition; roughly, it is an 'eventual stability' condition. See [22] for a full treatment; here we merely state the definition and consequence.

Definition 2.8 An inverse system $\left(A_{i}\right)_{i \in I}$, where $(I, \leq)$ is a totally ordered inverse system (not merely partially ordered) satisfies the Mittag-Leffler condition if for all $i$ there exists $j \geq i$ such that

$$
\operatorname{im}\left(A_{k} \rightarrow A_{i}\right)=\operatorname{im}\left(A_{j} \rightarrow A_{i}\right)
$$

for all $k \geq j$. That is, the images of the transition maps into $A_{i}$ are eventually stable.

If all systems $C_{n, i}(i \in I)$ in an inverse system of chain complexes $C_{\bullet, i}$ satisfy the MittagLeffler condition, then we will have

$$
\lim _{i} H_{n}\left(C_{\bullet, i}\right)=H_{n}\left(\lim _{i} C_{\bullet}, i\right)
$$

for all $n$. In our case, all the groups $C_{n, i}$ will be finite, so that the Mittag-Leffler condition holds (a decreasing sequence of subsets of a finite set is eventually constant). Our indexing set $I=\left\{(m, K) \mid m \in \mathbb{N}, K \leq_{\mathrm{f}} G\right\}$ will not be totally ordered; however by passing to the cofinal subset $J=\left\{\left(m !, K_{n}\right)\right\}$ where $K_{n}$ is the intersection of the finitely many subgroups of index at most $n$, we get a totally ordered indexing set without affecting the limit. 
Proposition 2.9 Let $\left(C_{i}\right)_{0 \leq i \leq n} \rightarrow \mathbb{Z}$ be a partial resolution of $\mathbb{Z}$ by free finitely generated $\mathbb{Z}[G]$-modules $C_{i}=\mathbb{Z}[G]^{\oplus r_{i}}$ where $G$ is a good group. Then $\left(\hat{C}_{i}\right)_{0 \leq i \leq n} \rightarrow \hat{\mathbb{Z}}$ is a partial resolution of $\hat{\mathbb{Z}}$ by free $\hat{\mathbb{Z}}[[\hat{G}]]$-modules

$$
\hat{C}_{i}=\hat{\mathbb{Z}}[[\hat{G}]]^{\oplus r_{i}}
$$

Proof For each $m \in \mathbb{N}$ and $K \leq_{\mathrm{f}} G$, set

$$
A_{i, m, K}=(\mathbb{Z} / m)[G / K] \otimes_{\mathbb{Z}[G]} C_{i}=(\mathbb{Z} / m)[G / K]^{\oplus r_{i}}
$$

so that the new chain groups are

$$
\hat{C}_{i}=\lim _{m, K} A_{i, m, K}
$$

The groups $A_{i, m, K}$ are finite, so the homology of each chain complex $\left(A_{\bullet}, m, K\right)$ is finite; as described above we may now use the Mittag-Leffler condition to conclude

$$
H_{i}\left(\hat{C}_{\bullet}\right)=H_{i}\left(\lim _{m, K} A_{i, m, K}\right)=\lim _{m, K} H_{i}\left(A_{i, m, K}\right)
$$

Regarding $\left(C_{\bullet}\right)$ as an exact complex of free finitely generated $\mathbb{Z}[K]$-modules and noting that

$$
A_{i, m, K}=(\mathbb{Z} / m) \otimes_{\mathbb{Z}[K]} C_{i}
$$

these homology groups $H_{i}\left(A_{i, m, K}\right)$ are precisely $H_{i}(K ; \mathbb{Z} / m)$. By the goodness of $G$ we can now use Proposition 2.7 to conclude

$$
H_{i}\left(\hat{C}_{\bullet}\right)=\lim _{m, K} H_{i}(K ; \mathbb{Z} / m)=0
$$

for $n-1 \geq i \geq 1 ;$ and for $i=0$

$$
H_{0}\left(\hat{C}_{\bullet}\right)=\lim _{m, K} H_{0}(K ; \mathbb{Z} / m)=\lim _{m, K} \mathbb{Z} / m=\hat{\mathbb{Z}}
$$

i.e. $\left(\hat{C}_{\bullet}\right)$ is a free partial resolution of $\hat{\mathbb{Z}}$.

\section{Profinite completions of 2-orbifold groups}

In this section we recall the results of Bridson et al. [6] concerning Fuchsian groups (i.e. orbifold fundamental groups of hyperbolic 2-orbifolds), and show that they extend to the case of Euclidean 2-orbifolds.

Theorem 3.1 (Theorem 1.1 of [6]) Let $G_{1}$ be a finitely-generated Fuchsian group and $G_{2}$ be a lattice in a connected Lie group. If $\hat{G}_{1} \cong \hat{G}_{2}$ then $G_{1} \cong G_{2}$.

Corollary 3.2 Let $O_{1}, O_{2}$ be closed 2-orbifolds. If $\pi_{1}^{\text {orb }\left(O_{1}\right)} \cong \pi_{1}^{\text {orb }\left(O_{2}\right)}$ then $\pi_{1}^{\text {orb }}\left(O_{1}\right) \cong$ $\pi_{1}^{\text {orb }}\left(O_{2}\right)$. If $\chi^{\text {orb }}\left(O_{1}\right) \leq 0$, then $O_{1}$ and $O_{2}$ are homeomorphic as orbifolds.

Proof Since $\pi_{1}^{\text {orb }}\left(O_{1}\right)$ is finite if and only if the orbifold Euler characteristic is positive, we can safely ignore these cases as the profinite completion is then simply the original group.

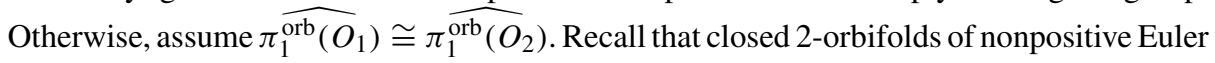
characteristic are determined by their fundamental groups. 
The orbifold has a finite cover which is a surface; take such a cover of $O_{1}$ and the corresponding cover of $\mathrm{O}_{2}$. If necessary pass to a further finite cover so that both $\mathrm{O}_{1}$ and $\mathrm{O}_{2}$ are covered with degree $d$ by surfaces with isomorphic profinite completions. A surface group is determined by its first homology, which is seen by the profinite completion, so the two surfaces are homeomorphic to the same surface $\Sigma$. Orbifold Euler characteristic is multiplicative under finite covers, so $\chi^{\text {orb }}\left(O_{1}\right)=\chi(\Sigma) / d=\chi^{\text {orb }}\left(O_{2}\right)$. Hence Euclidean and hyperbolic orbifolds are distinguished from each other.

It only remains, in light of the above theorem of Bridson-Conder-Reid, to distinguish the Euclidean 2-orbifolds from each other. The profinite completion detects first homology; a direct computation shows that this suffices to distinguish all the Euclidean 2-orbifolds except $\left(\mathbb{S}^{2} ; 2,4,4\right)$ and $\left(\mathbb{P}^{2} ; 2,2\right)$. Recall that an isomorphism of profinite completions would induce a correspondence between the index 2 subgroups, with corresponding subgroups having the same profinite completions. But $\left(\mathbb{P}^{2} ; 2,2\right)$ is covered by the Klein bottle with degree 2 , and the Klein bottle is distinguished from the other 2-orbifolds by its profinite completion, but does not cover $\left(\mathbb{S}^{2} ; 2,4,4\right)$. So these two Euclidean orbifolds also have distinct profinite completions.

Theorem 3.3 (Theorem 5.1 of [6]) Let $G$ be a finitely generated Fuchsian group. Every finite subgroup of $\hat{G}$ is conjugate to a subgroup of $G$, and if two maximal finite subgroups of $G$ are conjugate in $\hat{G}$ then they are already conjugate in $G$.

Proposition 3.4 Let $G$ be the fundamental group of a closed Euclidean 2-orbifold X. Every torsion element of $\hat{G}$ is conjugate to a torsion element of $G$, and if two torsion elements of $G$ are conjugate in $\hat{G}$ then they are already conjugate in $G$.

Proof The second statement is a special case of the fact that a virtually abelian group is conjugacy separable [21].

We proceed on a case-by-case basis. If $X$ is a torus or Klein bottle, then $G$ is good and has finite cohomological dimension, hence $\hat{G}$ has finite cohomological dimension and so is torsion free. If $X=\left(\mathbb{S}^{2} ; 2,2,2,2\right)$ then $G$ is the amalgamated free product of two copies of the infinite dihedral group. The result then holds by the same argument as in Theorem 5.1 of [6]; for a finite subgroup of the fundamental group of a graph of groups must be conjugate into one of the vertex groups, which here are the copies of $\mathbb{Z} / 2$. The same result holds profinitely. Similarly if $X=\left(\mathbb{P}^{2} ; 2,2\right)$ then the fundamental group is an amalgamated free product.

In [6] the triangle orbifolds were dealt with by passing to certain finite covers which decompose as amalgams, and whose fundamental group contains the torsion element of interest. However for Euclidean orbifolds, it may happen that no such covers exist; indeed no orbifold whose fundamental group is an amalgam has any cone points of order greater than 2. We will instead exploit the fact that our triangle groups are virtually abelian. We give in detail the proof for the orbifold $X=\left(\mathbb{S}^{2} ; 3,3,3\right)$; the other two triangle orbifolds are similar but involve checking more cases, so it would be uninformative to include the proofs.

Let $G=\left\langle a, b \mid a^{3}, b^{3},(a b)^{3}\right\rangle$. We have a short exact sequence

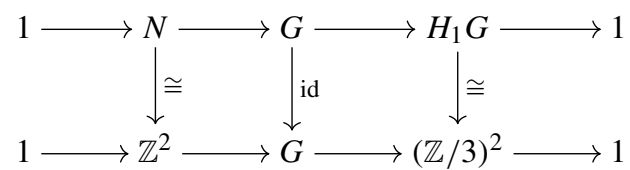


The subgroup $N$ is a subgroup of the translation subgroup of $G$. The translation subgroup is generated by the translations $x=a^{-1} b, y=b a^{-1}$. The action of conjugation is

$$
x^{a}=x^{b}=y^{-1} x^{-1}, \quad x^{a^{-1}}=x^{b^{-1}}=y \text { etc }
$$

The subgroup $N=\left\langle a b a^{-1} b^{-1}\right\rangle$ is then generated by the elements $u=y^{-1} x, v=x^{3}$; note that $[a, b]=u^{2} v^{-1}$. To guide our calculations, note that an element $a u^{r} v^{s}$ of $G$ acts on the plane by rotation about the centroid of a certain triangle, whose location is in fact that of the fixed point of the rotation $a$, translated by $u^{r+s} v^{r / 3}$. So in $G$, we have

$$
a u^{r} v^{s}=a^{u^{r+s} v^{r / 3}}=a^{y^{-r-s} x^{s}}
$$

and we expect similar equations to hold in $\hat{G}$.

We have a short exact sequence for $\hat{G}$ induced from the one above:

$$
1 \rightarrow \hat{\mathbb{Z}}^{2} \rightarrow \hat{G} \rightarrow(\mathbb{Z} / 3)^{2} \rightarrow 1
$$

and see that any torsion element of $\hat{G}$ is of the form $a^{i} b^{j} u^{\rho} v^{\sigma}$ where $i, j=0,1,2$ are not both zero and $\rho, \sigma \in \hat{\mathbb{Z}}$. For example, take $i=1, j=0$; the other cases are very similar. We now calculate

$$
\begin{aligned}
a^{y^{-\rho-\sigma} x^{\sigma}} & =x^{-\sigma} y^{\rho+\sigma} \cdot a \cdot y^{-\rho-\sigma} x^{\sigma} \\
& =a \cdot\left(x^{-\sigma} y^{\rho+\sigma}\right)^{a} y^{-\rho-\sigma} x^{\sigma} \\
& =a \cdot\left(y^{-1} x^{-1}\right)^{-\sigma} x^{\rho+\sigma} y^{-\rho-\sigma} x^{\sigma} \\
& =a \cdot y^{-\rho} x^{\rho} x^{3 \sigma}=a u^{\rho} v^{\sigma}
\end{aligned}
$$

So that torsion elements of this form are indeed conjugates of elements in $G$. The rest of the proof consists of similar calculations for other cases and can be safely omitted.

\section{Seifert fibre spaces}

We first recall some information about the invariants of a Seifert fibre space before moving on to profinite matters. For a more comprehensive introduction to Seifert fibre spaces see [8] and [19].

Recall that a fibred solid torus is formed as a quotient of $\mathbb{D}^{2} \times[0,1]$ by identifying the two end discs by a rotation by $2 \pi q / p$ where $p, q$ are coprime integers, called the fibre invariants of the fibred solid torus. The foliation of $\mathbb{D}^{2} \times[0,1]$ by lines $\{x\} \times[0,1]$ descends to a foliation of the torus by circles. Such pieces form a local model for a Seifert fibre space. Note that the quotient of a fibred solid torus obtained by collapsing each fibre naturally has an orbifold structure, where the image of the exceptional fibre is a cone point of order $p$. After fixing an orientation for the disc and fibre, the number $q$ becomes well-defined in the range $0<q<p$; if no orientations are chosen, it is well-defined only in the range $0<q \leq p / 2$. To give the standard presentation for the fundamental group, it is conventional to define the Seifert invariants of the exceptional fibre to be $(\alpha, \beta)$ where $\alpha=p$, and $\beta q \equiv 1 \bmod p$.

The orbifold quotients of neighbourhoods of each fibre piece together to form the quotient of the whole manifold $M$ by the foliation; this is the base orbifold $O$ of the Seifert fibre space. This quotient induces a short exact sequence

$$
1 \rightarrow<h>\rightarrow \pi_{1} M \rightarrow \pi_{1}^{\text {orb }} O \rightarrow 1
$$


where $\pi_{1}^{\text {orb }} O$ is the orbifold fundamental group, and $h$ is the element of $\pi_{1}$ represented by a regular fibre. This subgroup $\langle h\rangle$ may be finite or infinite cyclic, and is either central (if $O$ is orientable) or $\pi_{1} M$ has an index 2 subgroup which contains $h$ as a central element.

The final invariant has various different formulations; see [8,16,19]. It is in some sense the 'obstruction to a section', and coincides with the Euler number of the fibration when there are no exceptional fibres and the Seifert fibre space is a bona fide fibre bundle. In general it is still called the Euler number $e$ of the Seifert fibre space, and is a rational number. The key properties of the Euler number are the above behaviour when there are no exceptional fibres, and the following naturality property:

Proposition 4.1 If $\tilde{M} \rightarrow M$ is a degree d cover, where the base orbifold cover $\tilde{O} \rightarrow O$ has degree $m$ and a regular fibre of $\tilde{M}$ covers a regular fibre of $M$ with degree $l$

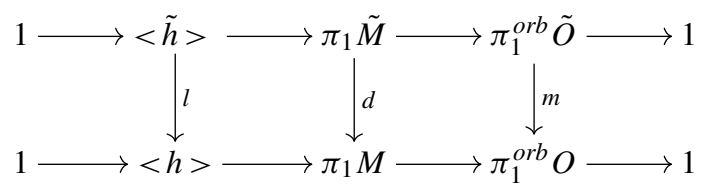

then

$$
e(\tilde{M})=\frac{m}{l} \cdot e(M)
$$

The Euler number has no well-defined sign a priori; given a choice of orientation on $M$, $e$ acquires a sign, and reversing the orientation (by flipping the direction along the fibres) changes this sign. This is consistent with the interpretation as the obstruction to a section; when there are no exceptional fibres, circle bundles with orientable total space are classified by elements of $H^{2}(\Sigma ; \mathbb{Z})$, where the $\mathbb{Z}$ coefficients are twisted by the orientation homomorphism for $\Sigma$; this group is $\mathbb{Z}$ whether or not $\Sigma$ is orientable.

The vanishing of the Euler number gives important topological information:

Proposition 4.2 Let $M$ be a Seifert fibre space. The Euler number e $(M)$ vanishes if and only if $M$ is virtually a surface bundle over the circle with periodic monodromy.

Finally, we can state the classification results of Seifert fibre spaces and characterisations of their fundamental groups from these invariants.

Proposition 4.3 A Seifert fibre space is uniquely determined by the symbol

$$
\left(b, \Sigma ;\left(\alpha_{1}, \beta_{1}\right), \ldots,\left(\alpha_{r}, \beta_{r}\right)\right)
$$

where

- $b \in \mathbb{Z}$ and $e=-\left(b+\sum \beta_{i} / \alpha_{i}\right)$;

$-\Sigma$ is the underlying surface of the base orbifold;

- $\left(\alpha_{i}, \beta_{i}\right)$ are the Seifert invariants of the exceptional fibres, and $0<\beta_{i}<\alpha_{i}$ are coprime.

If $\Sigma$ is closed and orientable of genus $g, \pi_{1} M$ has presentation

$$
\begin{aligned}
& \left\langle a_{1}, \ldots, a_{r}, u_{1}, v_{1}, \ldots, u_{g}, v_{g}, h\right| \\
& \left.\quad h \in Z\left(\pi_{1} M\right), a_{i}^{\alpha_{i}} h^{\beta_{i}}, a_{1} \ldots a_{r}\left[u_{1}, v_{1}\right] \cdots\left[u_{g}, v_{g}\right]=h^{b}\right\rangle
\end{aligned}
$$

If $\Sigma$ is closed and non-orientable of genus $g$, then $\pi_{1} M$ has presentation

$$
\begin{aligned}
& \left\langle a_{1}, \ldots, a_{r}, v_{1}, \ldots, v_{g}, h\right| \\
& \left.\quad h^{a_{i}}=h, h^{v_{i}}=h^{-1}, a_{i}^{\alpha_{i}} h^{\beta_{i}}, a_{1} \ldots a_{r} v_{1}^{2} \ldots v_{g}^{2}=h^{b}\right\rangle
\end{aligned}
$$


Table 1 The geometry of a Seifert fibre space is determined by the base orbifold and Euler number

\begin{tabular}{llll}
\hline & $\chi^{\text {orb }}>0$ & $\chi^{\text {orb }}=0$ & $\chi^{\text {orb }}<0$ \\
\hline$e=0$ & $\mathbb{S}^{2} \times \mathbb{R}$ & $\mathbb{E}^{3}$ & $\mathbb{H}^{2} \times \mathbb{R}$ \\
$e \neq 0$ & $\mathbb{S}^{3}$ & Nil & $\widehat{\mathrm{SL}_{2}(\mathbb{R})}$
\end{tabular}

When the Seifert fibre space has boundary, we have similar presentations without the last relation; the base orbifold group is just a free product of (finite or infinite) cyclic groups. In these presentations, $h$ represents the regular fibre; killing $h$ gives a presentation of the orbifold fundamental group of the base. Note also that reversing the orientation of the fibre $h$ and 'renormalising' to get the $\beta_{i}$ back into the correct range, there is an ambiguity in the above symbol for a Seifert fibre space, under the transformation

$$
\left(b, \Sigma ;\left(\alpha_{1}, \beta_{1}\right), \ldots,\left(\alpha_{r}, \beta_{r}\right)\right) \rightarrow\left(-b-r, \Sigma ;\left(\alpha_{1}, \alpha_{1}-\beta_{1}\right), \ldots,\left(\alpha_{r}, \alpha_{r}-\beta_{r}\right)\right)
$$

which flips the sign of $e$. When the orbifold is orientable, this will be the only ambiguity provided there is a unique Seifert fibre space structure on the manifold.

Proposition 4.4 If a closed manifold $M$ has two distinct Seifert fibre space structures, then it is covered by $\mathbb{S}^{3}, \mathbb{S}^{2} \times \mathbb{R}$, or $\mathbb{S}^{1} \times \mathbb{S}^{1} \times \mathbb{S}^{1}$.

Proposition 4.5 If $h$ is a regular fibre, then the subgroup $<h>$ is infinite cyclic unless $M$ is covered by $\mathbb{S}^{3}$.

Proposition 4.6 A manifold $M$ is Seifert fibred if and only if it has one of the six geometries in Table 1. The geometry is determined by the Euler characteristic of the base orbifold and the Euler number of $M$.

\section{Theorems}

In this section we prove the following result, which with the work of [24] gives the theorem in the introduction.

Theorem 5.1 Let $M_{1}, M_{2}$ be (closed orientable) Seifert fibre spaces. Then $\widehat{\pi_{1} M_{1}} \cong \widehat{\pi_{1} M_{2}}$ if and only if one of the following holds:

- $\pi_{1} M_{1} \cong \pi_{1} M_{2}$, so that unless they have $\mathbb{S}^{3}$-geometry, $M_{1}$ and $M_{2}$ are homeomorphic;

- $M_{1}, M_{2}$ have the geometry $\mathbb{H}^{2} \times \mathbb{R}$, where for some hyperbolic surface $S$ and some periodic automorphism $\phi$ of $S$, the 3-manifolds $M_{1}$ and $M_{2}$ are $S$-bundles over the circle with monodromy $\phi$ and $\phi^{\kappa}$ respectively, where $\kappa$ is coprime to $\operatorname{order}(\phi)$.

The non-trivial part of the 'if' direction of this theorem was proved by Hempel [13]. Alternatively one can apply the argument of Theorem 5.9 to get a new proof.

The solution of the problem will proceed in several stages. Firstly, we will show that, except in the 'trivial' geometries, an isomorphism of profinite completions of Seifert fibre spaces will induce an automorphism of the profinite completion of the base orbifold group $\hat{B}$, which the two Seifert fibre spaces will share; and furthermore that both Seifert fibre spaces will have the same Euler number (up to sign). We will then constrain the automorphism of $\hat{B}$ and compute the action of such an automorphism on $H^{2} \hat{B}$. Intuitively we will be considering 
what can happen to the 'fundamental class' of the orbifold. We will then be able to conclude the result by considering the cohomology classes giving the Seifert fibre spaces as central extensions of $\hat{B}$.

The 'trivial' geometries mentioned above are $\mathbb{S}^{3}, \mathbb{E}^{3}, \mathbb{S}^{2} \times \mathbb{R}$; they are trivial for the profinite rigidity problem in the sense that spherical manifolds have finite fundamental group, and there are only six and two orientable manifolds of the latter two geometries respectively, all distinguished by their first homology. For the rest of the section, a 'generic' Seifert fibre space will mean any Seifert fibre space not of the above geometries.

We will be using heavily the fact that the subgroup generated by a regular fibre is central; this is only true for orientable base orbifold, so first note that we can reduce to this case as follows. Suppose first that we have a closed Seifert fibre space. The orbifold group $B$ has a canonical index 2 subgroup corresponding to the orientation cover of the underlying surface of the orbifold. This induces an index 2 cover of the Seifert fibre space. Note that this cover contains all the information needed to recover the original Seifert fibre space; in particular, for each exceptional fibre with Seifert invariants $(p, q)$ where $1 \leq q<p / 2$ the cover has 2 exceptional fibres with the same invariant $(p, q)$, and has no other exceptional fibres. Because the index 2 subgroup is canonical, it will follow that any isomorphism of the profinite completions of the Seifert fibre space groups will induce an isomorphism for these characteristic covers, to which we may apply the theorem for orientable base orbifold, and then recover the original manifolds.

When the Seifert fibre space has boundary, the base orbifold group itself does not distinguish orientable base orbifold from non-orientable, and hence has no obvious characteristic subgroup. However if we assume that the peripheral subgroups of the base orbifold groups are preserved under the isomorphism of profinite completions, we can collapse each of them to obtain a closed orbifold and take the canonical index 2 cover of this, and hence of the original orbifold, to recover the above situation.

Throughout we will freely use the fact that Seifert fibre space groups and Fuchsian groups are subgroup separable. In particular if $M$ is a Seifert fibre space over a base orbifold $O$, we may take profinite completions to obtain a short exact sequence

$$
1 \rightarrow \widehat{\mathbb{Z}} \rightarrow \widehat{\pi_{1} M} \rightarrow \widehat{\pi_{1}^{\text {orb }} O} \rightarrow 1
$$

where $\hat{\mathbb{Z}}$ is generated by a regular fibre. these facts are theorems of Scott [18].

\subsection{Preservation of the fibre}

We first prove that the subgroup given by the fibre is still essentially unique for most Seifert fibre spaces. In the statement of the theorem, a 'virtually central' subgroup $Z$ of a group $G$ will mean that either $Z$ is central in $G$ or that the ambient group $G$ has an index 2 subgroup containing $Z$ in which $Z$ is central. The fibre subgroup of a Seifert fibre space subgroup is such a subgroup; it is central when the base orbifold is orientable, or is central in the index 2 subgroup corresponding to the orientation cover of a non-orientable base orbifold.

Theorem 5.2 Let $M, N$ be Seifert fibre spaces and suppose that $\widehat{\pi_{1}(M)} \cong \widehat{\pi_{1}(N)}$. Call this common completion $\Gamma$. Then:

1. $M$ and $N$ have the same geometry;

2. $\Gamma$ has a unique maximal virtually central normal procyclic subgroup unless the geometry of $M$ is $\mathbb{S}^{3}, \mathbb{S}^{2} \times \mathbb{R}$, or $\mathbb{E}^{3}$; and 
3. If the geometry is $\mathrm{Nil}, \mathbb{H}^{2} \times \mathbb{R}$, or $\widetilde{\mathrm{SL}_{2}(\mathbb{R})}$, then $M$ and $N$ have the same base orbifold and Euler number.

Remark 5.3 The first conclusion of this theorem was already known by the above-cited theorem of [24]; the proof here, specific to Seifert fibre spaces, is different in some respects, so we include it for completeness.

Proof As usual, spherical manifolds are distinguished by having finite fundamental groups, hence finite profinite completions. The four model geometries $\mathbb{E}^{3}, \mathrm{Nil}, \mathbb{H}^{2} \times \mathbb{R}$, and $\widetilde{\mathrm{SL}_{2}(\mathbb{R})}$ are contractible, so the fundamental groups of all such manifolds have cohomological dimension exactly 3. All compact $\mathbb{S}^{2} \times \mathbb{R}$-manifolds are finitely covered by $\mathbb{S}^{2} \times \mathbb{S}^{1}$, hence have a finite index subgroup of cohomological dimension 1. All Seifert fibre space groups are good, so this fact is detected by the profinite completion, hence $\mathbb{S}^{2} \times \mathbb{R}$ is distinguished from the other geometries. Henceforth assume that $M$ has one of the four relevant geometries with contractible universal cover.

Now suppose that $\Gamma$ has two virtually central normal procyclic subgroups, $\overline{\langle h\rangle}$ and $\overline{\langle\eta\rangle}$, where $h$ is represented by a regular fibre of $M$ and $\overline{\langle\eta\rangle}$ is not contained in $\overline{\langle h\rangle}$. We will show first that the base orbifold $O$ is Euclidean. Passing to the quotient by $\overline{\langle h\rangle}$, the image of $\overline{\langle\eta\rangle}$ is a normal procyclic subgroup of $\widehat{\pi_{1}^{\text {orb }}(O)}$. By Corollary 5.2 of [6] and Proposition 3.4 above, profinite completions of non-positively curved orbifold groups have no finite normal subgroups, so $\overline{\langle\eta\rangle}$ persists as an infinite procyclic subgroup of $\widehat{\pi_{1}^{\text {orb }}(O)}$. Hence also the subgroup $\overline{\langle h\rangle}$ is still maximal even in the profinite completion i.e. is not contained in some larger normal procyclic subgroup.

We can now pass to a finite index subgroup of $\Gamma$ whose intersections with $\overline{\langle h\rangle}, \overline{\langle\eta\rangle}$ are central and non-trivial, and then to a further finite index subgroup $\Delta$ so that the corresponding cover of $M$ has base orbifold an orientable surface $\Sigma$ covering $O$. The image of $\overline{\langle\eta\rangle}$ now gives a central subgroup of $\widehat{\pi_{1} \Sigma}$. But the profinite completion of a surface group has no centre unless the surface is a torus (see [2,15] or [3]). Hence $O$ is Euclidean.

The base orbifold $\Sigma$ is now a torus. We know that $\overline{\langle\eta\rangle}$ is a central procyclic subgroup of $\widehat{\pi_{1} \mathbb{T}^{2}} \cong \hat{\mathbb{Z}}^{2}$; assume now that it is maximal. Using Theorem 4.3.5 of [17], the quotient $\hat{\mathbb{Z}}^{2} /\langle\eta\rangle$ is $\hat{\mathbb{Z}}$; hence we can quotient by the closed subgroup $\hat{\mathbb{Z}}^{2}$ generated by both $h$ and $\eta$ to get an exact sequence

$$
1 \rightarrow \hat{\mathbb{Z}}^{2} \rightarrow \Delta \rightarrow \hat{\mathbb{Z}} \rightarrow 1
$$

We now calculate that

$$
H^{1}(\Delta ; \mathbb{Z} / n) \cong(\mathbb{Z} / n)^{3}
$$

for all $n$. As described in Sect. 2 we can calculate this cohomology group using the spectral sequence whose $E_{2}^{p, q}$ page is given by

$$
E_{2}^{p, q}=H^{p}\left(\hat{\mathbb{Z}} ; H^{q}\left(\hat{\mathbb{Z}}^{2} ; \mathbb{Z} / n\right)\right)
$$




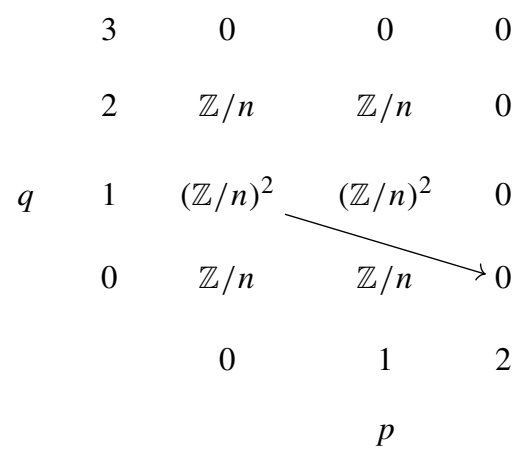

Now the only arrow that could alter the $p+q=1$ diagonal is the arrow shown, which is trivial; so this diagonal is already stable and the first cohomology is $(\mathbb{Z} / n)^{3}$ as required.

But the finite index subgroup $\Delta \leq \Gamma$ corresponds to a cover $\tilde{M} \rightarrow M$ where the base orbifold of $\tilde{M}$ is a torus. Then we have

$$
\left.\pi_{1} \tilde{M}=\left\langle u_{1}, v_{1}, h\right|\left[u_{1}, v_{1}\right]=h^{-e}, h \text { central }\right\rangle
$$

where $e$ is the Euler number of $\tilde{M}$; hence $H_{1} \tilde{M}=\mathbb{Z}^{2 g} \oplus \mathbb{Z} / e \mathbb{Z}$ and

$$
H^{1}(\Delta ; \mathbb{Z} / n) \cong H^{1}(\tilde{M} ; \mathbb{Z} / n) \cong(\mathbb{Z} / n)^{2} \oplus \mathbb{Z} / \operatorname{hcf}(e, n)
$$

for all $n$. Comparing with the above, we find that $e$ must be zero; by naturality $M$ also has trivial Euler number.

We now deal with the case where $\Gamma$ has a unique maximal virtually central procyclic normal subgroup. Note that in this case, the isomorphism $\widehat{\pi_{1}(M)} \cong \widehat{\pi_{1}(N)}$ preserves $\overline{<h>}$, and hence induces an isomorphism of the profinite completions of the base orbifold; then by Theorem 3.1 and Corollary 3.2, $M$ and $N$ have the same base orbifold $O$.

If we now show that $M, N$ have the same Euler number, then we are finished as the geometries are distinguished by base orbifolds and whether the Euler number is non-zero. Again pass to an index $d$ subgroup $\Delta$ of $\Gamma$ with the corresponding cover of $M$ being $\tilde{M} \rightarrow M$; where $M$ has base orbifold a surface. Then, as above, for both $N$ and $M$, the Euler number is given up to sign by the torsion part of $H_{1} \tilde{M}$, divided by $d$, because the Euler number has the naturality property in Proposition 4.1. First homology is a profinite invariant, hence $N$ and $M$ have the same Euler number and the proof is complete.

Recall that the Euler number of the Seifert fibre space was of the form

$$
e=-\left(b+\sum \frac{\beta_{i}}{\alpha_{i}}\right)
$$

with $b$ an integer. Thus given the base orbifold (hence the $\alpha_{i}$ ) and the Euler number, the only further ambiguity is whether we can change the $\beta_{i}$ by values $\delta_{i}$ (with $\delta_{i}$ not congruent to 0 modulo $\alpha_{i}$ ) such that $\sum \delta_{i} / \alpha_{i}$ is an integer. By the Chinese Remainder Theorem, there is no such collection of $\delta_{i}$ when all the $\alpha_{i}$ are coprime. Hence we have the following corollary, in which we change notation to follow the usual conventions for cone points.

Corollary 5.4 Let $M$ be a Seifert fibre space whose base orbifold is an orbifold

$$
\left(\Sigma ; p_{1}, \ldots, p_{k}\right)
$$


where $p_{1}, \ldots, p_{k}$ are coprime. Then $\pi_{1} M$ is distinguished by its profinite completion from all other 3-manifold groups.

The above theorem was stated and proved for closed Seifert fibre spaces. A similar result holds for Seifert fibre spaces with boundary. Much of the above argument holds just as well when the Seifert fibre space has boundary, except that we must rule out some cases with more than one geometry, and the Euler number is no longer defined (a section of a surface-withboundary always exists). Furthermore, surfaces are no longer determined by their profinite completion unless we have some information about the boundary.

Theorem 5.5 Let $M, N$ be Seifert fibre spaces with non-empty boundary. Suppose that $\widehat{\pi_{1}(M)} \cong \widehat{\pi_{1}(N)}$. Call this common completion $\Gamma$. Furthermore assume that $M$ and $N$ have the same number of boundary components. Then:

1. $\Gamma$ has a unique maximal virtually central normal procyclic subgroup unless $M$ (and hence $N$ ) is a solid torus, $\mathbb{S}^{1} \times \mathbb{S}^{1} \times \mathbb{I}$ or the orientable $\mathbb{I}$-bundle over the Klein bottle; and

2. except in these cases, $M$ and $N$ have the same base orbifold.

Proof The only positive Euler characteristic orbifolds with boundary are the disc with possibly one cone point; the Seifert fibre space is then a fibred solid torus.

The only zero Euler characteristic orbifolds with boundary are the annulus (giving the Seifert fibre space $\mathbb{S}^{1} \times \mathbb{S}^{1} \times \mathbb{I}$ ), the Möbius band and disc with two order 2 cone points (both giving the orientable $\mathbb{I}$-bundle over the Klein bottle).

These three spaces all have different profinite completions of fundamental groups; one is $\hat{\mathbb{Z}}$, one is $\hat{\mathbb{Z}}^{2}$ and the other is non-abelian; and none of the Seifert fibre spaces with hyperbolic base orbifold have virtually abelian fundamental group, so we can safely proceed assuming $M, N$ are not any of the three exceptional manifolds above.

Part 1 of the proposition now follows from the same argument as in Theorem 5.1, replacing "virtually a non-abelian surface group" with "virtually a non-abelian free group" to get the lack of central subgroups of the base orbifold group. Now the base orbifold groups have isomorphic profinite completions, so by [6], they are the same group. The ambiguity in the surface is now resolved by the knowledge that $M$ and $N$ and hence their base orbifolds have the same number of boundary components; and the fact that $\Gamma$ detects whether the unique maximal virtually central normal subgroup $<h>$ is genuinely central or merely virtually so, hence whether the base orbifold is orientable or not.

\subsection{Central extensions}

A central extension of a group $B$ by a (necessarily abelian) group $A$ consists of a short exact sequence

$$
1 \rightarrow A \rightarrow G \rightarrow B \rightarrow 1
$$

where the image of $A$ is contained in the centre of $G$. Two such extensions are regarded as equivalent if there is a commutative diagram

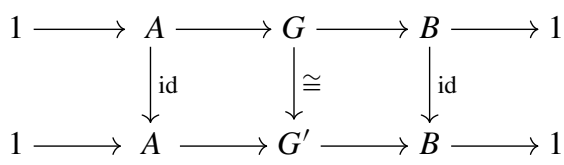


Equivalence classes of central extensions are classified by elements of $H^{2}(B ; A)$. The proof of this fact proceeds directly via cochains, but for what follows it will also be convenient to have the following interpretation.

Let $B=\left\langle x_{1}, \ldots, x_{n} \mid r_{1}, \ldots, r_{m}\right\rangle$ be a presentation for $B$, let $F$ be the free group on the $x_{i}$, and $R$ the normal subgroup generated by the $r_{j}$. From the Serre spectral sequence for the short exact sequence

$$
1 \rightarrow R \rightarrow F \rightarrow B \rightarrow 1
$$

we obtain the five-term exact sequence

$$
0 \rightarrow H^{1}(B ; A) \rightarrow H^{1}(F ; A) \rightarrow\left(H^{1}(R ; A)\right)^{F} \rightarrow H^{2}(B ; A) \rightarrow 0=H^{2}(F ; A)
$$

where the third non-zero term denotes those elements of $H^{1}(R ; A)$ invariant under the conjugation action of $F$; in fact this is the group $H^{1}(R /[R, F] ; A)$. Given an element of $H^{2}(B ; A)$, lift to some

$$
\xi \in\left(H^{1}(R ; A)\right)^{F}=(\operatorname{Hom}(R, A))^{F}
$$

Then a central extension of $B$ by $A$ is given by the 'presentation' (abusing notation slightly):

$$
G=\left\langle Y, x_{1}, \ldots, x_{n} \mid S, Y \subseteq Z(G), r_{1}=\xi\left(r_{1}\right), \ldots, r_{m}=\xi\left(r_{m}\right)\right\rangle
$$

where $A=\langle Y \mid S\rangle$. The condition that $A$ does genuinely include into this group is equivalent to the invariance of $\xi$ under the action of $F$. The ambiguity under choice of lift to an element $\xi$ is an element $\psi \in H^{1}(F ; A)$. However this ambiguity corresponds precisely to changing the generating set of $G$ to $Y$ and the elements $x_{i}^{\prime}=x_{i} \cdot \psi\left(x_{i}\right)$. Conversely if two such $G, G^{\prime}$ given by $\xi, \xi^{\prime}$ are isomorphic by an isomorphism $\Phi$ fixing $B$ and $A$, then $\xi$ and $\xi^{\prime}$ differ by $\psi \in H^{1}(F ; A)$ given by $\psi\left(x_{i}\right)=x_{i} \cdot\left(\Phi\left(x_{i}\right)\right)^{-1}$.

The question of when two central extensions $G, G^{\prime}$ of $B$ by $A$ given by $\zeta, \zeta^{\prime} \in H^{2}(B ; A)$ can be isomorphic allowing arbitrary automorphisms for $B$ and $A$ is more subtle; one needs to prove whether any automorphisms of $B$ and $A$ can carry $\zeta$ to $\zeta^{\prime}$ by the induced maps on $H^{2}$. This will be the central issue in the proof of Theorem 5.1.

The above theory of central extensions also holds for $\hat{B}$ profinite, provided that the abelian group $A$ is finite so that the cohomology group $H^{2}(\hat{B}, A)$ is reasonably well-behaved. See Sect. 6.8 of [17]. The fundamental groups of generic Seifert fibre spaces (over orientable base) are central extensions

$$
1 \rightarrow \mathbb{Z} \rightarrow G \rightarrow B \rightarrow 1
$$

classified by an element $\eta_{G} \in H^{2}(B ; \mathbb{Z})$, where $B=\pi_{1}^{\text {orb }} O$ is the fundamental group of the base orbifold. The profinite completion of a generic Seifert fibre space group is a central extension of $\hat{B}$ by the infinite group $\hat{\mathbb{Z}}$. To avoid the complications raised by the presence of $\hat{\mathbb{Z}}$, we restrict to a finite coefficient group as follows. Note that since an isomorphism of profinite completions of two Seifert fibre space groups $G, G^{\prime}$ preserves this central subgroup $\hat{\mathbb{Z}}$ by Theorem 5.2 , and since $\hat{\mathbb{Z}}$ has a unique index $t$ subgroup, any isomorphism $\hat{G} \cong \hat{G}^{\prime}$ induces an isomorphism

$$
\Gamma=\hat{G} / \overline{<h^{t}>} \cong \hat{G}^{\prime} / \overline{<h^{t}>}=\Gamma^{\prime}
$$

where $\Gamma, \Gamma^{\prime}$ are now central extensions of $\hat{B}$ by $\mathbb{Z} / t$. Hence they are classified by elements $\zeta, \zeta^{\prime}$ of $H^{2}(\hat{B} ; \mathbb{Z} / t)$. But $B$ is a good group, hence $H^{2}(\hat{B} ; \mathbb{Z} / t)$ is canonically isomorphic to $H^{2}(B ; \mathbb{Z} / t)$; and $\zeta_{G}, \zeta_{G^{\prime}}$ are the images of $\eta_{G}, \eta_{G^{\prime}}$ under the maps

$$
H^{2}(B ; \mathbb{Z}) \rightarrow H^{2}(B ; \mathbb{Z} / t) \cong H^{2}(\hat{B} ; \mathbb{Z} / t)
$$


It remains to show that no automorphisms of $\hat{B}$ and $\mathbb{Z} / t$ can carry $\zeta_{G}$ to $\zeta_{G^{\prime}}$ under the induced maps on $H^{2}(\hat{B} ; \mathbb{Z} / t)$ for all $t$ unless the manifolds $M_{1}, M_{2}$ are homeomorphic or are covered by the theorem of Hempel [13].

Before moving on, let us calculate the cohomology classes $\eta_{G}$ in terms of the five-term exact sequence; this will be of use later. For a Seifert fibre space over orientable base with symbol

$$
\left(b, \Sigma ;\left(p_{1}, q_{1}\right), \ldots,\left(p_{r}, q_{r}\right)\right)
$$

the fundamental group has presentation $\left\langle a_{1}, \ldots, a_{r}, u_{1}, v_{1}, \ldots, u_{g}, v_{g}, h\right|$

$$
\left.h \in Z\left(\pi_{1} M\right), a_{i}^{p_{i}} h^{q_{i}}, a_{1} \ldots a_{r}\left[u_{1}, v_{1}\right] \ldots\left[u_{g}, v_{g}\right]=h^{b}\right\rangle
$$

Let $1 \rightarrow R \rightarrow F \rightarrow B \rightarrow 1$ be the corresponding presentation of the base orbifold group. Now $R /[R, F]$ is in fact the free $\mathbb{Z}$-module on these relations $y_{0}=a_{1} \ldots v_{g}^{-1}, y_{i}=a_{i}^{p_{i}}$; comparing to the above general theory we see that the cohomology class $\eta_{G}$ is the image in $H^{2}(B ; \mathbb{Z})$ of the map

$$
y_{0} \mapsto b, \quad y_{i} \mapsto-q_{i}
$$

in $\operatorname{Hom}(R /[R, F], A)$. The chain complexes in the following section make rigorous our treatment of $R /[R, F]$ as a free abelian group on these generators. The calculation is similar for the bounded case, except that the $y_{0}$ term does not appear.

\subsection{Action on cohomology}

We first constrain the possible automorphisms of base orbifold that we need to consider:

Proposition 5.6 Let $M_{1}, M_{2}$ be generic Seifert fibre spaces with $\widehat{\pi_{1}\left(M_{1}\right)} \cong \widehat{\pi_{1}\left(M_{2}\right)}$. Let the base orbifold group be

$$
B=\left\langle a_{1}, \ldots, a_{r}, u_{1}, v_{1} \ldots, u_{g}, v_{g} \mid a_{1}^{p_{1}}, \ldots, a_{r}^{p_{r}}, a_{1} \ldots a_{r} \cdot\left[u_{1}, v_{1}\right] \cdots\left[u_{g}, v_{g}\right]\right\rangle
$$

Then some isomorphism of $\widehat{\pi_{1}\left(M_{1}\right)}$ with $\widehat{\pi_{1}\left(M_{2}\right)}$ induces an automorphism of $\hat{B}$ mapping each $a_{i}$ to a conjugate of $a_{i}^{k_{i}}$, where $k_{i}$ is coprime to $p_{i}$.

Proof This is a simple corollary of Proposition 3.4; for the induced automorphism of $\hat{B}$ from any given isomorphism of the $\widehat{\pi_{1}\left(M_{i}\right)}$ must induce a bijection on conjugacy classes of maximal torsion elements; hence $a_{i}$ is sent to a conjugate of $a_{\sigma(i)}^{k_{i}}$ for some permutation $\sigma$ with $p_{\sigma(i)}=p_{i}$ and $k_{i}$ coprime to $p_{i}$. Permuting the $a_{i}$ under the permutation $\sigma^{-1}$ is an automorphism of $B$, hence of $\hat{B}$, so we can force $\sigma$ to be the identity; on the level of the Seifert fibre spaces we are simply relabelling the exceptional fibres and exploiting the invariance of the fundamental group under such relabellings.

Note that this proposition works just as well when there is boundary.

Proposition 5.7 If $\phi$ is an automorphism of $B$ as in Proposition 5.6, then for any $n$ the action of $\phi^{*}$ on $H^{2}(\hat{B} ; \mathbb{Z} / n)$ is multiplication by $\kappa$ for some profinite integer $\kappa \in \hat{\mathbb{Z}}$ such that for all $1 \leq i \leq r, \kappa$ is congruent to $k_{i}$ modulo $p_{i}$. 
Proof We construct a partial resolution of $\mathbb{Z}$ by free $\mathbb{Z} B$-modules, transport this to a partial resolution of $\hat{\mathbb{Z}}$ by free $\hat{\mathbb{Z}}[[\hat{B}]]$-modules, and use this to compute the action on cohomology of the above automorphisms of $B$. Fix the presentation

$$
B=\left\langle a_{1}, \ldots, a_{r}, u_{1}, v_{1} \ldots, u_{g}, v_{g} \mid a_{1}^{p_{1}}, \ldots, a_{r}^{p_{r}}, a_{1} \cdots a_{r} \cdot\left[u_{1}, v_{1}\right] \cdots\left[u_{g}, v_{g}\right]\right\rangle
$$

of $B$, let $F=F\left(a_{i}, u_{i}, v_{i}\right)$ and $R=\operatorname{ker}(F \rightarrow B)$.

Set $C_{0}=\mathbb{Z} B$, interpreted as the free $\mathbb{Z}$-module on the vertices of the Cayley graph of $B$, with $B$-action by left translation on $\operatorname{Cay}(B)$; the map $\epsilon: \mathbb{Z} B \rightarrow \mathbb{Z}$ is the evaluation map.

Let $C_{1}=\mathbb{Z} B\left\{x_{i}, \bar{u}_{j}, \bar{v}_{j}\right\}$, the free $\mathbb{Z} B$-module with generators $x_{i}(1 \leq i \leq r), \bar{u}_{j}, \bar{v}_{j}(1 \leq$ $j \leq g$ ). The generator $x_{i}$ represents the edge in Cay $(B)$ starting at 1 and labelled by $a_{i}$, and similarly $\bar{u}_{j}, \bar{v}_{j}$ represent the edges labelled $u_{j}, v_{j}$. Thus $C_{1}$ is the space of linear combinations of paths in $\operatorname{Cay}(B)$, with $B$-action given by left-translation.

The boundary map $d_{1}: C_{1} \rightarrow C_{0}$ sends each path to the sum of its endpoints, so that for example $x_{i} \mapsto a_{i}-1 \in \mathbb{Z} B$. Certainly $\epsilon d_{1}=0$; exactness at $C_{0}$ now follows by connectedness of the Cayley graph.

Let $C_{2}=\mathbb{Z} B\left\{y_{0}, \ldots, y_{r}\right\}$. We can interpret $C_{2}$ as representing 'all the relations of $B$ '; that is, all closed loops in the Cayley graph. The generator $y_{0}$ will represent the relation $a_{1} \cdots v_{g}^{-1}$ in the above presentation, and $y_{i}$ the relation $a_{i}^{p_{i}}$; now define $d_{2}: C_{2} \rightarrow C_{1}$ by mapping each generator to the loop in the Cayley graph representing it; for instance,

$$
\begin{aligned}
d_{2}\left(y_{i}\right)= & x_{i}+a_{1} \cdot x_{i}+a_{1}^{2} \cdot x_{i}+\cdots+a_{i}^{p_{i}-1} \cdot x_{i} \\
d_{2}\left(y_{0}\right)= & x_{1}+a_{1} \cdot x_{2}+\cdots+a_{1} \ldots a_{r-1} \cdot x_{r} \\
& +a_{1} \ldots a_{r} \cdot \overline{u_{1}}+\cdots-a_{1} \ldots a_{r}\left[u_{1}, v_{1}\right] \cdots\left[u_{g}, v_{g}\right] \bar{v}_{g}
\end{aligned}
$$

Any loop in the Cayley graph represents some element of $R$, which can be expressed as a product of conjugates of the relations in the above presentation. Left conjugation of a relation corresponds to left-translating the loop around the Cayley graph; so any such product of conjugates can be realised in the Cayley graph as a $\mathbb{Z} B$-linear combination of the $d_{2}\left(y_{i}\right)$. Hence $d_{1} d_{2}=0$ and the image of $d_{2}$ is precisely the kernel of $d_{1}$.

Let us analyse the kernel of $d_{2}$; let

$$
s=\sum_{i} \sum_{b} n_{b}^{i} b \cdot y_{i} \in \operatorname{ker}\left(d_{2}\right)
$$

where $\sum_{b} n_{b}^{i} b \in \mathbb{Z} B$ for each $i$. The coefficient of $x_{i}$ in $d_{2}(s)$ is

$$
0=\sum_{b} n_{b}^{0} b a_{1} \ldots a_{i-1}+\sum_{b} n_{b}^{i} b\left(1+a_{i}+\cdots a_{i}^{p_{i}-1}\right)
$$

Multiplying on the right by $\left(a_{i}-1\right)$ kills the second sum; and reparametrising the first sum yields $n_{b a_{i}}^{0}=n_{b}^{0}$ for all $b \in B$. If $r>1$, the $a_{i}$ generate an infinite subgroup of $B$; but $\sum n_{b}^{0} b$ is a finite linear combination, so $n_{b}^{0}=0$ for all $b$. If $r=1$, we can analyse the coefficient of $u_{i}$ instead as $g>0$ for a non-spherical orbifold; or we can simply note that profinite rigidity in the cases $r=0,1$ was already covered by Corollary 5.4, so that we need not worry any further about them. We are left to conclude that $\sum_{b} n_{b}^{i} b\left(1+a_{i}+\cdots a_{i}^{p_{i}-1}\right)=0$, hence $\sum_{b} n_{b}^{i} b$ is some multiple of $\left(a_{i}-1\right)$ and the kernel of $d_{2}$ is spanned by $\left(a_{i}-1\right) y_{i}$.

Now set $C_{3}=\mathbb{Z} B\left\{z_{1}, \ldots, z_{r}\right\}$ and $d_{3}\left(z_{i}\right)=\left(a_{i}-1\right) \cdot y_{i}$ to find an exact sequence

$$
C_{3} \rightarrow C_{2} \rightarrow C_{1} \rightarrow C_{0} \rightarrow \mathbb{Z}
$$

i.e. a partial resolution of $\mathbb{Z}$ by free $\mathbb{Z} B$-modules as desired. 
By Proposition 2.9 we have a partial resolution

$$
\hat{C}_{3} \rightarrow \hat{C}_{2} \rightarrow \hat{C}_{1} \rightarrow \hat{C}_{0} \rightarrow \hat{\mathbb{Z}}
$$

where each $\hat{C}_{i}$ is the free $\hat{\mathbb{Z}}[[\hat{B}]]$-module on the same generators as $C_{i}$, and the boundary maps are defined by the same formulae on these generators. We can thus use this resolution to compute the first and second (co)-homology on $\hat{B}$.

Let $\phi: \hat{B} \rightarrow \hat{B}$ be an automorphism of $\hat{B}$ as in Proposition 5.6. Construct maps $\phi_{\sharp}$ : $\hat{C}_{i} \rightarrow \hat{C}_{i}$ for $i=1,2$ as follows. Lift $\phi$ to $\tilde{\phi}: \hat{F} \rightarrow \hat{F}$ such that

$$
\tilde{\phi}\left(a_{i}\right)=\left(a_{i}^{k_{i}}\right)^{g_{i}^{-1}}
$$

for some $g_{i} \in \hat{F}$. Write the image of each generator of $\hat{F}$ under $\tilde{\phi}$ as a limit of words on these generators; then map the corresponding generator of $\hat{C}_{1}$ to the associated limit of paths in the Cayley graph. To define $\phi_{\sharp}$ on $\hat{C}_{2}$, note that each relation of $\hat{B}$ is mapped to an element of $\bar{R}$ under $\tilde{\phi}$, hence can be written as a (limit of) products of conjugates of relations; now map this to an element of $\hat{C}_{2}$ just like before. We have made a choice of expression of an element of $\bar{R}$ in terms of conjugates of relations; the ambiguity is by construction an element of $\operatorname{ker}\left(\hat{d}_{2}\right)=\operatorname{im}\left(\hat{d}_{3}\right)$, which image will soon vanish. For definiteness, choose

$$
\phi_{\sharp}\left(y_{i}\right)=k_{i} g_{i} \cdot y_{i} \quad(1 \leq i \leq r)
$$

coming from the obvious expression of $\tilde{\phi}\left(a_{i}^{p_{i}}\right)$ from above. Because the map on $\bar{R}$ was induced by the map on $\hat{F}$ used to define $\phi_{\sharp}: \hat{C}_{1} \rightarrow \hat{C}_{1}$, we get a commuting diagram

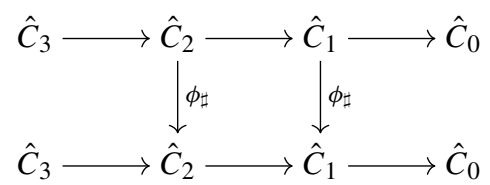

Now apply the functor $\hat{\mathbb{Z}} \otimes_{\hat{\mathbb{Z}}[[\hat{B}]]}$-to the above diagram; i.e., factor out the action of $\hat{B}$, to get a commuting diagram

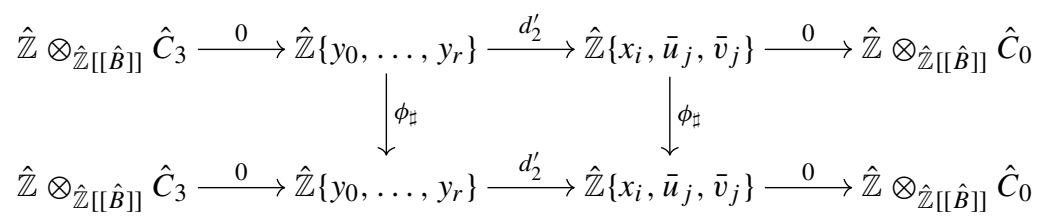

with the rows no longer exact, but with the maps marked as zero becoming trivial because the image of each generator of the chain group had a factor $\left(a_{i}-1\right)$. We have some good control over the maps in the above, viz.

$$
\begin{aligned}
& \phi_{\sharp}\left(x_{i}\right)=k_{i} x_{i} \\
& \phi_{\sharp}\left(y_{i}\right)=k_{i} y_{i} \\
& d_{2}^{\prime}\left(y_{0}\right)=x_{1}+\cdots+x_{r} \\
& d_{2}^{\prime}\left(y_{i}\right)=p_{i} x_{i}
\end{aligned}
$$

If $\phi_{\sharp}\left(y_{0}\right)=\kappa y_{0}+\sum \mu_{i} y_{i}$, then tracking this around the diagram we find

$$
\kappa+p_{i} \mu_{i}=k_{i}
$$

for all $i$. 
For $n \in \mathbb{N}$, we now apply $\operatorname{Hom}_{\hat{\mathbb{Z}}}(-, \mathbb{Z} / n)$ to the above diagram, to get a commuting diagram

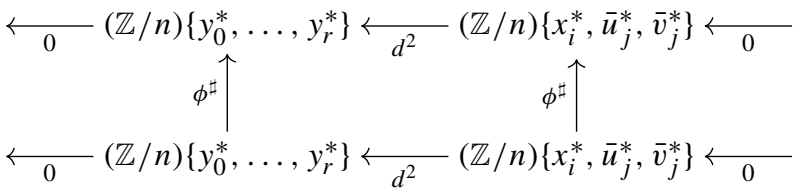

in which the homology of each row gives $H^{2}(\hat{B} ; \mathbb{Z} / n)$ and $\phi^{\sharp}$ gives an action on this cohomology group.

First let us note that this action is genuinely the functorial map $\phi^{*}$ induced by $\phi$. By construction $\hat{\mathbb{Z}} \otimes_{\hat{\mathbb{Z}}[[\hat{B}]]} \hat{C}_{2}$ is the free $\hat{\mathbb{Z}}$-module on our relations. In this construction for the discrete group, this would be $R /[R, F]$. In the profinite world, $\bar{R} /[\bar{R}, \hat{F}]$ may not be free abelian, as not every closed subgroup of a free profinite group is free; however we do get a canonical surjection

$$
\hat{\mathbb{Z}} \otimes_{\hat{\mathbb{Z}}[[\hat{B}]]} \hat{C}_{2} \rightarrow \bar{R} /[\bar{R}, \hat{F}]
$$

since our chosen set of relations is a generating set for this latter group. But now the map $\phi_{\sharp}$ on $\hat{\mathbb{Z}} \otimes_{\hat{\mathbb{Z}}[[\hat{B}]]} \hat{C}_{2}$ is easily seen to induce the natural map on $\bar{R} /[\bar{R}, \hat{F}]$ given by $\tilde{\phi}$; and naturality of the quotient map

$$
H^{1}(\bar{R} /[\bar{R}, \hat{F}] ; \mathbb{Z} / n) \rightarrow H^{2}(\hat{B} ; \mathbb{Z} / n)
$$

coming from the five-term exact sequence shows that $\phi^{\sharp}$ will indeed give the correct action on $H^{2}$.

Finally, we can compute this action on $H^{2}(\hat{B} ; \mathbb{Z} / n)$. We have from above

$$
\begin{aligned}
& \phi^{\sharp}\left(y_{0}^{*}\right)=\kappa y_{0}^{*} \\
& \phi^{\sharp}\left(y_{i}^{*}\right)=\mu_{i} y_{0}^{*}+k_{i} y_{i}^{*} \\
& d^{2}\left(x_{i}^{*}\right)=y_{0}^{*}+p_{i} y_{i}^{*} \\
& d^{2}\left(\bar{u}_{i}^{*}\right)=0=d^{2}\left(\bar{v}_{i}^{*}\right)
\end{aligned}
$$

so that, given a cochain $\zeta=b y_{0}^{*}-\sum_{i} q_{i} y_{i}^{*}$, we have

$$
\begin{aligned}
\phi^{*}([\zeta])=\left[\phi^{\sharp}(\zeta)\right] & =\left[\left(\kappa b-\sum q_{i} \mu_{i}\right) y_{0}^{*}-\sum q_{i} k_{i} y_{i}^{*}\right] \\
& =\left[\kappa\left(b y_{0}^{*}-\sum q_{i} y_{i}^{*}\right)-\sum q_{i} \mu_{i}\left(y_{0}^{*}+p_{i} y_{i}^{*}\right)\right] \\
& =\kappa[\zeta]
\end{aligned}
$$

Proof (Proof of Theorem 5.1) Recall that we have reduced to the case of orientable base orbifold. As discussed in Sect. 5.2, our manifolds $M_{1}, M_{2}$ are determined by cohomology classes $\eta_{1}, \eta_{2} \in H^{2}(B ; \mathbb{Z})$. If

$$
M_{1}=\left(b, \Sigma ;\left(p_{1}, q_{1}\right), \ldots,\left(p_{r}, q_{r}\right)\right)
$$


then as a cochain in the basis $y_{0}^{*}, \ldots, y_{r}^{*}$ of $\operatorname{Hom}_{\mathbb{Z} B}\left(C_{2}, \mathbb{Z}\right)$ where $C_{\bullet}$ is the partial resolution defined above, we have (see Sect. 5.2)

$$
\eta_{1}=\left[b y_{0}^{*}-\sum_{1 \leq i \leq r} q_{i} y_{i}^{*}\right]
$$

and similarly for $\eta_{2}$. From these we get cohomology classes $\zeta_{i, n} \in H^{2}(\hat{B} ; \mathbb{Z} / n)$. Suppose that $\widehat{\pi_{1}\left(M_{1}\right)} \cong \widehat{\pi_{1}\left(M_{2}\right)}$. Then, after possibly reordering the exceptional fibres of $M_{2}$, we have that the exists $\kappa \in \hat{\mathbb{Z}}$ such that $\kappa \zeta_{1, n}=\zeta_{2, n}$ for all $n$. It is a consequence of the previous proposition that an automorphism of the base induces such an effect on the cohomology groups; we may also rescale the fibres of the $M_{i}$ by an automorphism of $\hat{\mathbb{Z}}$, giving an automorphism of the coefficient ring of $H^{2}$. But this is simply multiplication of the cohomology class by some element of $\hat{\mathbb{Z}}^{\times}$, which we merge into $\kappa$.

If the $M_{i}$ have non-zero Euler number $e>0$ (by reversing the orientation on the fibres we can always force $e>0$ for both manifolds), choose $n=m e \prod p_{i}$ for some integer $m$, and define a group homomorphism $E: H^{2}(\hat{B} ; \mathbb{Z} / n) \rightarrow \mathbb{Z} / n$ by

$$
E\left(\sum t_{i} y_{i}^{*}\right)=-t_{0} \prod p_{j}+\sum_{i \neq 0} t_{i} \prod_{j \neq i} p_{j}
$$

so that $E(\kappa \xi)=\kappa E(\xi)$. Since $e=-\left(b+\sum q_{i} / p_{i}\right)$, we have $E\left(\zeta_{1, n}\right)=e \prod p_{j}$ modulo $n$; then

$$
E\left(\kappa \zeta_{1, n}-\zeta_{2, n}\right)=(\kappa-1) e \prod p_{j}=0 \text { modulo } n
$$

so that $\kappa \zeta_{1, n}=\zeta_{2, n}$ for all $n=m e \prod p_{j}$ can only hold if $\kappa$ is congruent to 1 modulo $m$ for all $m$, i.e. if $\kappa=1$ and $\zeta_{1, n}=\zeta_{2, n}$ for all $n$, so that $\eta_{1}=\eta_{2}$ and $M_{1}, M_{2}$ are homeomorphic.

If the $M_{i}$ have Euler number zero, so that they are $\mathbb{H}^{2} \times \mathbb{R}$ manifolds, choose $n=\prod p_{i}$ and $k \in \mathbb{Z}$ such that $k$ is congruent to $\kappa \bmod n$. Then $M_{2}$ is a Seifert fibre space with zero Euler characteristic and Seifert invariants $\left(p_{i}, k q_{i}\right)$; there is only one such, and Hempel showed that these pairs of $\mathbb{H}^{2} \times \mathbb{R}$ manifolds are precisely those surface bundles in the statement of the theorem.

Rather easier is the bounded case, given sensible conditions on the boundary.

Theorem 5.8 Let $M_{1}, M_{2}$ be orientable Seifert fibre spaces with boundary, and assume that there exists an isomorphism $\Phi: \widehat{\pi_{1} M_{1}} \rightarrow \widehat{\pi_{1} M_{2}}$ inducing an isomorphism of peripheral systems, in the following sense. The boundary components of $M_{1}$ determine a conjugacy class of $\mathbb{Z}^{2}$-subgroups in the fundamental group, which gives a conjugacy class of $\hat{\mathbb{Z}}^{2}$-subgroups in the profinite completion. The isomorphism $\Phi$ is required to send one such set of conjugacy classes to the other, inducing isomorphisms on the matched copies of $\hat{\mathbb{Z}}^{2}$.

Let $M_{1}$ have Seifert invariants $\left(p_{i}, q_{i}\right)$. Then for some $k \in \mathbb{Z}$ coprime to all $p_{i}, M_{2}$ is the Seifert fibre space with the same base orbifold and Seifert invariants $\left(p_{i}, k q_{i}\right)$.

Proof We can safely focus on hyperbolic base orbifolds, the other three Seifert fibre spaces with boundary being easily distinguished from these and each other by their first homology, hence by the profinite completion. As before, we have already reduced to the case of orientable base orbifold.

Note that two boundary components of the base orbifolds generate distinct free $\hat{\mathbb{Z}}$ factors of the base orbifold group, and the standard theory of free profinite products (see Theorem 9.1.12 of [17]) shows that these are not conjugate in the profinite completion; so the number 
of peripheral conjugacy classes remains the same as the number of boundary components. Then by Theorem 5.5 both Seifert fibre spaces share the same base orbifold $O$, and there is an induced automorphism of $\hat{B}=\widehat{\pi_{1}^{\mathrm{orb}} O}$. As before, we can now consider the Seifert fibre spaces as being represented by elements of $H^{2}(\hat{B} ; \mathbb{Z} / n)$ for arbitrary $n$.

Take a presentation

$$
B=\left\langle a_{1}, \ldots, a_{r}, b_{1}, \ldots, b_{s}, u_{1}, v_{1}, \ldots, u_{g}, v_{g} \mid a_{1}^{p_{1}}, \ldots, a_{r}^{p_{r}}\right\rangle
$$

for the base orbifold, where the $a_{i}$ are the cone points and the (conjugacy classes of the) $b_{i}$ give all but one of the boundary components; the remaining boundary component is

$$
b_{0}=a_{1} \ldots a_{r} \cdot b_{1} \ldots b_{s} \cdot\left[u_{1}, v_{1}\right] \ldots\left[u_{g}, v_{g}\right]
$$

As before, we are at liberty to permute cone points with the same order, and permuting boundary components is also permitted. Thus given Proposition 5.6 and the conditions of the theorem we may assume that the automorphism $\phi$ of $\hat{B}$ induced by $\Phi$ is of the form

$$
a_{i} \mapsto\left(a_{i}^{k_{i}}\right)^{g_{i}}, b_{j} \mapsto\left(b_{j}^{l_{j}}\right)^{h_{j}}
$$

for elements $g_{i}, h_{j}$ of $\hat{B}, l_{j} \in \hat{\mathbb{Z}}^{\times}$, and $k_{i}$ coprime to $p_{i}$.

Now the induced automorphism of

$$
H_{1}(\hat{B})=\hat{B}_{\mathrm{ab}}=\bigoplus_{i=1}^{r} \mathbb{Z} / p_{i} \oplus \bigoplus_{j=1}^{s} \hat{\mathbb{Z}} \oplus \hat{\mathbb{Z}}^{2 g}
$$

sends the class of $b_{0}$ to

$$
\phi_{*}\left(\left[b_{0}\right]\right)=\phi_{*}\left(\sum\left[a_{i}\right]+\sum_{j \neq 0}\left[b_{j}\right]\right)=\sum k_{i}\left[a_{i}\right]+\sum_{j \neq 0} l_{j}\left[b_{j}\right]
$$

and on the other hand to

$$
l_{0}\left[b_{0}\right]=\sum l_{0}\left[a_{i}\right]+\sum_{j \neq 0} l_{0}\left[b_{j}\right]
$$

showing that all the $k_{i}$ are congruent to $l_{0}$ modulo $p_{i}$ and that all the $l_{i}$ are equal.

Using essentially the same chain complex as in the closed case we can now compute that the action on

$$
H^{2}\left(\hat{B} ; \mathbb{Z} / \prod p_{i}\right)=\bigoplus_{i=1}^{r} \mathbb{Z} / p_{i}
$$

is multiplication by $l_{0}$, or equivalently multiplication by some $k \in \mathbb{Z}$ congruent to $l_{0}$ modulo $\prod p_{i}$, thus taking the element $\left(q_{1}, \ldots, q_{r}\right)$ representing $M_{1}$ to the element representing $M_{2}$, which we now see to be $\left(k q_{1}, \ldots, k q_{r}\right)$.

We finally prove the converse to the last theorem. A mild adjustment to this argument, with the appropriate modification of the cohomology group considered, provides another proof of Hempel's theorem on closed Seifert fibre spaces.

Theorem 5.9 Let $M_{1}, M_{2}$ be Seifert fibre spaces with non-empty boundary and with the same base orbifold $O$. Suppose $M_{1}$ has Seifert invariants $\left(p_{i}, q_{i}\right)$ and $M_{2}$ has Seifert invariants $\left(p_{i}, k q_{i}\right)$ where $k$ is some integer coprime to every $p_{i}$. Then $\widehat{\pi_{1} M_{1}} \cong \widehat{\pi_{1} M_{2}}$. 
Proof Again it suffices to deal with the case of orientable base orbifold. Let $\Gamma_{i}=\widehat{\pi_{1} M_{i}}$, let $h_{i}$ be a generator of the centre of $\pi_{1} M_{i}$, and let

$$
\Gamma_{i, n}=\Gamma_{i} / \overline{\left.<h^{t}\right\rangle}
$$

where $t=n \prod p_{i}$.

Note that for each $i$ the $\Gamma_{i, n}$ form a natural inverse system with maps $\Gamma_{i, n m} \rightarrow \Gamma_{i, n}$. Furthermore, any map from $\Gamma_{i}$ to a finite group must kill some power of $h$, and hence factors through some $\Gamma_{i, n}$. It follows that

$$
\Gamma_{i}=\lim _{n} \Gamma_{i, n}
$$

Now $k$ maps to an invertible element of $\mathbb{Z} / \prod p_{i}$; then there is some invertible element $\kappa$ of $\hat{\mathbb{Z}}$ congruent to $k$ modulo each $p_{i}$. One can prove this by noting that by the Chinese Remainder theorem the natural map $(\mathbb{Z} / m n)^{\times} \rightarrow(\mathbb{Z} / n)^{\times}$is always surjective, hence so is the map $\hat{\mathbb{Z}}^{\times} \rightarrow(\mathbb{Z} / n)^{\times}$.

As discussed in Sect. 5.2, $\Gamma_{i, n}$ is classified by an element

$$
\zeta_{i} \in H^{2}(\hat{B} ; \mathbb{Z} / t)=\bigoplus_{j=1}^{r} \mathbb{Z} / p_{j}
$$

where $B$ is the base orbifold group; by assumption $\zeta_{2}=k \zeta_{1}=\kappa \zeta_{1}$. Multiplication of the coefficient group $\kappa$ gives an automorphism of the cohomology group taking $\zeta_{1}$ to $\zeta_{2}$, hence induces an isomorphism $\Gamma_{1, n} \rightarrow \Gamma_{2, n}$. Moreover this isomorphism is compatible with the quotient maps $\Gamma_{i, n m} \rightarrow \Gamma_{i, n}$; hence we have an isomorphism

$$
\Gamma_{1}=\lim _{n} \Gamma_{1, n} \cong \lim _{n} \Gamma_{2, n}=\Gamma_{2}
$$

as required.

Open Access This article is distributed under the terms of the Creative Commons Attribution 4.0 International License (http://creativecommons.org/licenses/by/4.0/), which permits unrestricted use, distribution, and reproduction in any medium, provided you give appropriate credit to the original author(s) and the source, provide a link to the Creative Commons license, and indicate if changes were made.

\section{References}

1. Agol, I.: The virtual Haken conjecture. Doc. Math. 18, 1045-1087 (2013)

2. Anderson, M.P.: Exactness properties of profinite completion functors. Topology 13(3), 229-239 (1974)

3. Asada, M.: On centerfree quotients of surface groups. Commun. Algebra 29(7), 2871-2875 (2001)

4. Aschenbrenner, M., Friedl, S., Wilton, H.: 3-Manifold Groups. European Mathematical Society, Zurich (2015)

5. Boileau, M., Friedl, S.: The profinite completion of 3-manifold groups, fiberedness and the Thurston norm. arXiv preprint arXiv:1505.07799 (2015)

6. Bridson, M. R., Conder, D.E., Reid, A. W.: Determining Fuchsian groups by their finite quotients. arXiv preprint arXiv:1401.3645 (2014)

7. Bridson, M.R., Reid, A.W.: Profinite rigidity, fibering, and the figure-eight knot. arXiv preprint arXiv:1505.07886 (2015)

8. Brin, M.G.: Seifert fibered spaces: notes for a course given in the spring of 1993. arXiv preprint arXiv:0711.1346 (2007)

9. Cartan, H., Eilenberg, S.: Homological Algebra, vol. 19. Princeton University Press, Princeton (1999) 
10. Cavendish, W.P.: Finite-sheeted covering spaces and solenoids over 3-manifolds. PhD Thesis, Princeton (2012)

11. Funar, L.: Torus bundles not distinguished by TQFT invariants. Geom.Topol. 17(4), 2289-2344 (2013)

12. Grunewald, F., Jaikin-Zapirain, A., Zalesskii, P.A.: Cohomological goodness and the profinite completion of Bianchi groups. Duke Math. J. 144(1), 53-72 (2008)

13. Hempel, J.: Some 3-manifold groups with the same finite quotients. arXiv preprint arXiv:1409.3509 (2014)

14. Lackenby, M.: Finding disjoint surfaces in 3-manifolds. Geom. Dedicata. 170(1), 385-401 (2014)

15. Nakamura, H.: Galois rigidity of pure sphere braid groups and profinite calculus. J. Math. Sci. Univ. Tokyo 1, 71-136 (1994)

16. Neumann, W.D., Raymond, F.: Seifert manifolds, plumbing, $\mu$-invariant and orientation reversing maps. In: Algebraic and Geometric Topology. Lecture Notes in Mathematics, vol. 664, pp. 163-196. Springer (1978)

17. Ribes, L., Zalesskii, P.: Profinite Groups. Springer, Berlin (2000)

18. Scott, P.: Subgroups of surface groups are almost geometric. J. Lond. Math. Soc. 2(3), 555-565 (1978)

19. Scott, P.: The geometries of 3-manifolds. Bull. Lond. Math. Soc. 15(5), 401-487 (1983)

20. Serre, J.-P.: Galois cohomology. Springer, Berlin (2013)

21. Stebe, P.F.: Conjugacy separability of certain Fuchsian groups. Trans. Am. Math. Soc. 163, 173-188 (1972)

22. Weibel, C.A.: An Introduction to Homological Algebra. Number 38. Cambridge university press, Cambridge (1995)

23. Wilton, H., Zalesskii, P.: Profinite properties of graph manifolds. Geom. Dedicata. 147(1), 29-45 (2010)

24. Wilton, H., Zalesskii, P.: Distinguishing geometries using finite quotients. arXiv preprint arXiv: 1411.5212 (2014) 